\title{
Subsequent pregnancy outcomes in recurrent miscarriage patients with a paternal or maternal carrier of a structural chromosome rearrangement
}

\author{
Mayumi Sugiura-Ogasawara $\cdot$ Koji Aoki $\cdot$ Tomoyuki Fujii $\cdot$ Tomio Fujita \\ Rie Kawaguchi - Tetsuo Maruyama - Nobuaki Ozawa - Toshitaka Sugi · \\ Toshiyuki Takeshita $\cdot$ Shigeru Saito
}

Received: 18 September 2007 / Accepted: 20 March 2008/Published online: 15 April 2008

(C) The Japan Society of Human Genetics and Springer 2008

\begin{abstract}
Information concerning the prognosis of subsequent pregnancies in patients with reciprocal translocations is limited. This study was performed to determine the percentage success rate with first pregnancies after ascertainment of a carrier status. A total of 2,382 couples with a history of two or more consecutive miscarriages were studied in multicenters. The prevalence of an abnormal chromosome in either partner was examined, and subsequent success rates were compared between cases with and without an abnormal karyotype in either partner. A total of 129 couples (5.4\%) had an abnormal karyotype in one partner excluding inversion 9 in 44 men and in 85 women. Thus, 2,253 couples had a normal karyotype in both partner. Eighty-five (3.6\%) had translocations, 13 being Robertsonian translocations. Twenty-nine of the 46
\end{abstract}

M. Sugiura-Ogasawara ( $\square)$

Department of Obstetrics and Gynecology, Nagoya City

University Medical School, Mizuho-ku, Nagoya 467, Japan

e-mail: og.mym@med.nagoya-cu.ac.jp

K. Aoki

Aoki Ladies Clinic, Nagoya, Japan

T. Fujii

Department of Obstetrics and Gynecology,

Faculty of Medicine, University of Tokyo,

Tokyo, Japan

T. Fujita

Department of Maternal Medicine,

Osaka Prefectural Hospital Organization,

Osaka Medical Center and Research Institute for Maternal and Child Health, Izumi, Japan

R. Kawaguchi

Department of Obstetrics and Gynecology, Jikei University

School of Medicine, Tokyo, Japan cases $(63.0 \%)$ who became pregnant with reciprocal translocations in either partner experienced a live birth with natural conception. In contrast, 950 of 1,207 cases (78.7\%) with normal chromosomes had successful live births, the difference being significant $(P=0.019)$. No infant with an unbalanced translocation was found in 29 cases of successful pregnancy following recurrent miscarriage. Pregnancy prognosis was worsened with either maternal or paternal reciprocal translocations. Explanation of the success rate with natural conception should be provided before the subsequent pregnancy after ascertainment of carrier status.

Keywords Chromosome abnormality · Inversion · Recurrent miscarriage - Reciprocal translocation . Robertsonian translocation

T. Maruyama

Department of Obstetrics and Gynecology,

Keio University School of Medicine, Tokyo, Japan

N. Ozawa

Department of Perinatal Medicine and Maternal Care,

National Center for Child Health and Development,

Tokyo, Japan

T. Sugi

Department of Obstetrics and Gynecology, Specialized Clinical

Science, Tokai University School of Medicine, Kanagawa, Japan

T. Takeshita

Department of Obstetrics and Gynecology,

Nippon Medical School, Tokyo, Japan

S. Saito

Department of Obstetrics and Gynecology,

University of Toyama, Toyama, Japan 


\section{Introduction}

An abnormal karyotype in either partner, especially when a translocation is involved, is considered to be the cause of recurrent miscarriage (RM) (De Braekeleer and Dao 1990). De Braekeleer et al. analyzed a computerized database covering 22,199 couples generated from the literature on cytogenetic studies and concluded a rate of $4.7 \%$ for chromosomal structural rearrangements in couples suffering two or more spontaneous abortions.

The number of centers performing preimplantation genetic diagnosis (PGD) worldwide has been steadily increasing since the procedure's introduction over a decade ago (Handyside et al. 1990). Munne et al. (2000) concluded that PGD could achieve a statistically significant reduction in the miscarriage rate from $95 \%$ to $13 \%$ in translocation carriers. However, as most RM patients visit hospital because they experience difficulty in having children, it is inappropriate to compare miscarriage rates before and after diagnosis in RM cases. To our knowledge, there have been no case-control studies comparing live-birth rates between PGD and natural pregnancies after parents are diagnosed as carriers of translocations. Thus, it is unclear whether PGD can improve the birth rate in patients with translocations, although it does prevent miscarriages.

It is difficult to conduct case-control studies because translocation carriers are relatively rare. Recently, several manuscripts concerning reproductive outcome after natural conception in RM patients with a parental carrier of a structural chromosome rearrangement have been published (Sugiura-Ogasawara et al. 2004; Carp et al. 2004; Goddijn et al. 2004; Stephenson and Sierra 2006; Franssen et al. 2006). Sugiura-Ogasawara's 2004 study indicated a success rate of about $31.9 \%$ (15 of 47) at the first pregnancy after the ascertainment of carrier status, which is much less than that with normal chromosomes $(71.7 \%, 849$ of 1,184$)$, and a cumulative success rate of $68.1 \%$ (32 of the 47). They concluded that the prognosis of RM patients with reciprocal translocations is poor, given that the study was conducted over 17 years and included severe cases suffering ten and 13 miscarriages.

Recently, Franssen et al. (2006) reported cumulative success rates for RM patients with reciprocal translocations, Robertsonian translocations, and a normal karyotype to be $83.0 \%, 82.0 \%$, and $84.1 \%$, respectively, from their prospective case-control study. They thus concluded that the chance of having a healthy child is as high as in noncarrier couples, despite the higher risk of miscarriage.

However, available information on the prognosis of RM patients with a structural chromosome rearrangement is insufficient. This study therefore focused on success rate at the first pregnancy after ascertainment of carrier status.

\section{Patients and methods}

This multicenter study was performed in Nagoya City Johsai Hospital, Tokyo University Hospital, Osaka Medical Center and Research Institute for Maternal and Child Health, National Center for Child Health and Development, Toyama University Hospital, Tokai University Hospital, Nagoya City University Hospital, Nippon Medical School Hospital, Jikei University Hospital, and Keio University Hospital. Totally, 2,382 couples (4,764 individuals) with a history of two or more consecutive miscarriages who visited the hospitals between January 2003 and December 2005 were enrolled.

Hysterosalpingography, chromosome analysis for both partners, identification of antiphospholipid antibodies (aPL) such as lupus anticoagulant and $\beta 2$-glycoprotein-I-dependent anticardiolipin antibodies or anticardiolipin antibodies, and blood tests for hyperthyroidism, diabetes mellitus and hyperprolactinemia were performed for all cases before subsequent pregnancy. Their first pregnancies after ascertainment of carrier status were followed up till September 2007. Patients with at least one kind of aPL were treated with combined low-dose aspirin and heparin therapy. Interventions such as supportive psychotherapy were added to patients with both abnormal and normal karyotypes. Gestational age was calculated from basal body temperature charts. Dilation and curettage was performed when miscarriages were diagnosed, and the karyotypes of aborted conceptuses were ascertained with the use of a standard Gbanding technique. Informed consent was obtained from all patients. Informed consent for the multicenter study was approved by the institutional review board in Nagoya City University.

In our study:

1. The frequencies of abnormal karyotypes in either partner in Japan were examined.

2. The subsequent success rates were compared between cases with reciprocal translocation and with a normal karyotype. Miscarriage rates for patients with a Robertsonian translocation or inversions were also assessed.

Statistical analysis

Differences in group values were analyzed using Stat view with an Apple Macintosh computer. A significance level of $P<0.05$ was applied for all tests.

\section{Results}

1. A total of 129 of 2,382 couples $(5.4 \%)$ had an abnormal karyotype in one partner excluding inversion 9: 44 
were in men and 85 were in women. Seventy-two $(3.0 \%)$ had reciprocal translocations: 28 in men and 44 in women. In addition, 13 had Robertsonian translocations (seven in men and six in women). Thus, the overall frequency of translocations was $3.6 \%$. Other inversions existed in chromosomes 4,7 , and 8: in eight men; and 1, 2, 8, and 11: in 17 women. Twenty-six had low-frequency mosaicisms. Thus, 2,253 couples had a normal karyotype in both partner.

2. Subsequent pregnancy outcomes for reciprocal translocation carriers and details are shown in Tables 1 and 2. Forty-six women were found to be pregnant by natural conception after $10.1 \pm 7.7$ months from the ascertainment of reciprocal translocation carrier status. Twenty-nine (63.0\%) experienced a live birth. No infant with an unbalanced translocation was found in 29 cases of successful pregnancy following RM.

In contrast, of 1,207 women with a normal karyotype who became pregnant, $950(78.7 \%)$ had a successful live birth. The live birth rate in cases with reciprocal translocations was significantly lower than that for cases with normal chromosomes in both partners $(P=0.019)$. The mean age of translocation carriers $(31.0 \pm 3.9)$ at the diagnosis of carrier status was lower than that in cases with normal chromosomes $(32.9 \pm 4.3, P=0.0032)$. There were no differences in mean numbers of previous miscarriages between reciprocal translocation carriers $(3.1 \pm 1.2)$ and patients with normal chromosomes $(2.8 \pm 1.1, P=0.071)$. Twenty-nine $(2.4 \%)$ of the 1,207 control patients had uterine anomalies (14 bicornis, nine septum, three unicornis, three didelphys), and $26(2.2 \%)$ had at least one kind of aPL. None of the 46 pregnant reciprocal translocation carriers had uterine anomalies and aPLs.

When cases with only two previous miscarriages were excluded, 23 of the 34 women $(67.6 \%)$ experienced a live birth. When cases with a history of live birth were excluded, the figure was $62.5 \%$ (25/40). Three of the five cases $(60.0 \%)$ who had a Robertsonian translocation were included in those who demonstrated a live birth subsequently (Table 3).

Details for all pregnancy outcomes after examination of the 18 couples who had other abnormal chromosomes such

Table 1 Subsequent first pregnancy outcome in recurrent miscarriage couples

\begin{tabular}{ll}
\hline Parental karyotype & Live birth rates \\
\hline Reciprocal translocation & $29 / 46(63.0 \%)$ \\
Robertsonian translocation & $3 / 5(60.0 \%)$ \\
Inversion & $5 / 7(71.4 \%)$ \\
Low-frequency mosaicism & $9 / 17(52.9 \%)$ \\
Normal & $950 / 1207(78.7 \%)$ \\
\hline
\end{tabular}

as inversions are shown in Table 4. Five of the seven cases with inversions $(71.4 \%)$ could give birth to live babies. We included 26 cases $(1.09 \%)$ with low-frequency mosaicisms. Nine of the 17 women $(52.9 \%)$ could have living babies.

\section{Discussion}

In this study, 129 couples (5.4\%) had an abnormal karyotype in one partner excluding pericentric inversion of chromosome 9. The frequency is in line with previous studies (De Braekeleer et al. 1990), although it was $7.8 \%$ in Sugiura-Ogasawara's study because inversion 9, which is a normal variant, was included (Sugiura-Ogasawara et al. 2004). Reciprocal translocation is the most important problem in RM cases. Translocations are also found in infertile men, and thus, the frequency in women would be higher than that in men in RM cases after natural selection (Elghezal et al. 2006).

Five manuscripts concerning prospective reproductive outcome in RM patients with a parental carrier of a structural chromosome rearrangement have been published (Sugiura-Ogasawara et al. 2004; Carp et al. 2004; Goddijn et al. 2004; Stephenson and Sierra 2006; Franssen et al. 2006). Carp et al. examined the first pregnancy outcome after ascertainment of translocation carriers including Robertsonian translocations and described 19 of 44 (43.2\%) carriers to feature live births. Franssen et al. conducted a case-control study and prospectively followed up patients for a mean of 5.8 years by telephone. They found cumulative success rates for RM patients with reciprocal translocations, Robertsonian translocations, and a normal karyotype to be $83.0 \%, 82.0 \%$, and $84.1 \%$, respectively. Generally, RM patients tend not to be followed up after examination in University Hospitals because of distances from their home towns. Indeed, in our study, a certain number of patients did not visit each hospital after examination, presumably when they did not conceive or the subsequent pregnancy was followed up in another hometown hospital. Thus, Franssen's conclusions have an important bearing not only for RM patients with translocations but also those with normal chromosomes. Whereas the success rate of patients with translocations at the first pregnancy after ascertainment of carrier status could not be obtained, that with all kinds of carriers was $62.0 \%(148 / 239)$.

Regarding success rates at the first pregnancy after ascertainment of reciprocal translocation carrier status, this study, Stephenson and Sierra' study (2006), and SugiuraOgasawara et al.'s earlier study (2004) generated figures of $63.0 \%, 65.0 \%(13 / 20)$, and $31.9 \%$, respectively. The reason the prognosis of Sugiura-Ogasawara's patients was so poor is that the study included severe cases with large numbers 
Table 2 Carriers of a reciprocal translocation with a history of recurrent miscarriage

\begin{tabular}{|c|c|c|c|c|c|}
\hline Reciprocal translocation & Age & $\begin{array}{l}\text { Previous } \\
\text { miscarriage } \\
\text { (Stillbirth) }\end{array}$ & $\begin{array}{l}\text { Previous live } \\
\text { birth }\end{array}$ & $\begin{array}{l}\text { Pregnancy } \\
\text { outcome }\end{array}$ & Chromosome \\
\hline \multicolumn{6}{|l|}{ Female } \\
\hline $46, \mathrm{XX}, \mathrm{t}(1 ; 4)(\mathrm{q} 42.1 ; \mathrm{p} 15.32)$ & 39 & $3(1)$ & 0 & Not available ${ }^{a}$ & \\
\hline $46, X X, t(1 ; 5)(q 12 ; q 22)$ & 29 & 3 & 0 & Not available & \\
\hline $46, \mathrm{XX}, \mathrm{t}(1 ; 10)(\mathrm{q} 21 ; \mathrm{p} 11.2)$ & 34 & 4 & 0 & Failure & Not tested \\
\hline $46, X X, t(1 ; 10)(q 42.1 ; q 24.3)$ & 28 & 2 & 0 & Failure & Not tested \\
\hline $46, X X, t(1 ; 11)(p 11 q 13)$ & Not available & 2 & 0 & Failure & $47, \mathrm{XY},+4$ \\
\hline $46, \mathrm{XX}, \mathrm{t}(1 ; 15)(\mathrm{q} 32.1 ; \mathrm{q} 23)$ & 28 & 3 & 0 & Success & \\
\hline $46, \mathrm{XX}, \mathrm{t}(2 ; 12)(\mathrm{q} 36 ; \mathrm{p} 13.2)$ & 34 & 2 & 0 & Not conceive & \\
\hline $46, \mathrm{XX}, \mathrm{t}(2 ; 15)(\mathrm{p} 23 ; \mathrm{q} 15)$ & 23 & 3 & 0 & Success & \\
\hline $46, \mathrm{XX}, \mathrm{t}(2 ; 15)(\mathrm{q} 31 ; \mathrm{q} 21.2)$ & 38 & 6 & 0 & Success & \\
\hline 46,XX,t(2;18)(q33;p11.3) & 42 & 3 & 0 & Failure & Not tested \\
\hline 46,XX,t(3;5)(p13;q33) & 27 & 3 & 0 & Success & \\
\hline $46, X X, t(3: 7)(p 25 ; p 13)$ & 33 & 3 & 0 & Success & \\
\hline $46, \mathrm{XX}, \mathrm{t}(3 ; 9)(\mathrm{p} 13 ; \mathrm{q} 34)$ & 27 & 2 & 0 & Not available & \\
\hline $46, \mathrm{XX}, \mathrm{t}(3 ; 16)(\mathrm{q} 13.2 ; \mathrm{q} 22)$ & 35 & 2 & 0 & Not available & \\
\hline $\begin{array}{l}\text { 46,XX,inv(9)(p11p13), } \\
\mathrm{t}(4 ; 12)(\mathrm{q} 33 ; \mathrm{q} 23)\end{array}$ & 39 & 4 & 2 & Success & \\
\hline $46, \mathrm{XX}, \mathrm{t}(4 ; 21)(\mathrm{p} 15.1 ; \mathrm{q} 22.2)$ & 31 & 2 & 0 & Success & \\
\hline $46, X X, t(4 ; 5)(q 23 ; q 33.3)$ & 33 & 2 & 0 & Not conceive & \\
\hline $46, \mathrm{XX}, \mathrm{t}(5 ; 13)(\mathrm{p} 15.3 ; \mathrm{q} 21.2)$ & 33 & 3 & 0 & Success & \\
\hline $46, \mathrm{XX}, \mathrm{t}(6 ; 7)(\mathrm{q} 25.1 ; \mathrm{p} 21)$ & 28 & 3 & 0 & Failure & $46, X Y, \operatorname{der}(6) \mathrm{t}(6 ; 7)(\mathrm{q} 25.1 ; \mathrm{p} 21)$ \\
\hline $46, \mathrm{XX}, \mathrm{t}(6 ; 8)^{\mathrm{b}}$ & 33 & $5(1)$ & 0 & Failure & 46,XXdel(6)(q23) \\
\hline $46, X X, t(6 ; 8)(q 23 ; p 23)$ & 35 & $6(1)$ & 0 & Failure & $46, \mathrm{XX}, \mathrm{t}(6 ; 8)(\mathrm{q} 23 ; \mathrm{p} 23)$ \\
\hline $46, \mathrm{XX}, \mathrm{t}(6 ; 20)(\mathrm{q} 22.3 ; \mathrm{p} 13)$ & 30 & 2 & 0 & PGD failure & Not tested \\
\hline $46, \mathrm{XX}, \mathrm{t}(7 ; 8)(\mathrm{q} 11.2 ; \mathrm{q} 13)$ & 35 & 3 & 0 & Not available & \\
\hline 46,XX,t(7;11)(p13;q21) & 26 & 2 & 0 & Success & \\
\hline 46,XX,t(7;18)(p14;p11) & 41 & 4 & 0 & Not conceive & \\
\hline $46, \mathrm{XX}, \mathrm{t}(7 ; 18)(\mathrm{p} 15.3 ; \mathrm{p} 11.32)$ & 33 & 3 & 0 & Failure & Not tested \\
\hline $46, \mathrm{XX}, \mathrm{t}(7 ; 18)(\mathrm{q} 32 ; \mathrm{q} 13)$ & 38 & 4 & 0 & Failure & Not tested \\
\hline $46, \mathrm{XX}, \mathrm{t}(8 ; 10)(\mathrm{q} 13 ; \mathrm{q} 11.2)$ & 30 & 4 & 0 & Not available & \\
\hline $46, \mathrm{XX}, \mathrm{t}(9 ; 11)(\mathrm{q} 34.1 ; \mathrm{q} 23.1)$ & 29 & 5 & 1 & Not available & \\
\hline $46, \mathrm{XX}, \mathrm{t}(9 ; 13)(\mathrm{q} 12 ; \mathrm{p} 12)$ & 32 & 4 & 0 & Success & \\
\hline $46, \mathrm{XX}, \mathrm{t}(10 ; 16)(\mathrm{q} 26.3 ; \mathrm{p} 11.2)$ & 25 & 2 & 0 & Not conceive & \\
\hline $46, X X, t(10 ; 17)(q 26 ; p 12)$ & 28 & 3 & 0 & Failure & $46, X X, \operatorname{der}(17) t(10 ; 17)(\mathrm{q} 26 ; \mathrm{p} 12) \mathrm{mat}$ \\
\hline $46, \mathrm{XX}, \mathrm{t}(10 ; 21)(\mathrm{p} 10 ; \mathrm{q} 10)$ & 27 & 4 & 0 & Success & $46, X Y, t(10 ; 21)(\mathrm{p} 10 ; \mathrm{q} 10)$ \\
\hline $46, \mathrm{XX}, \mathrm{t}(11 ; 22)(\mathrm{q} 23.3 ; \mathrm{q} 11.2)$ & 28 & 2 & 0 & Success & \\
\hline $46, X X, t(11 ; 22)(\mathrm{q} 23 ; \mathrm{q} 11.2)$ & 29 & 3 & 0 & Failure & $46, \mathrm{XX}[25] / 46, \mathrm{XX}, \operatorname{del}(5)(\mathrm{p} 14)[5]$ \\
\hline $46, \mathrm{XX}, \mathrm{t}(11 ; 22)(\mathrm{q} 23.3 ; \mathrm{q} 11.2)$ & 27 & 3 & 0 & Not conceive & \\
\hline $46, \mathrm{XX}, \mathrm{t}(12 ; 21)(\mathrm{q} 13.3 ; \mathrm{q} 22.1)$ & 23 & 3 & 0 & Not conceive & \\
\hline $46, \mathrm{XX}, \mathrm{t}(13 ; 19)(\mathrm{q} 14 ; \mathrm{p} 13.1)$ & 31 & 2 & 0 & Not available & \\
\hline $46, X X, t(16 ; 20)(\mathrm{p} 11 ; \mathrm{p} 13)$ & 37 & 3 & 0 & Not available & \\
\hline $46, X X, t(17 ; 20)(p 13 ; q 13.1)$ & 31 & 3 & 1 & Failure & Not tested \\
\hline $46, X X, t^{b}$ & 26 & 2 & 0 & Failure & $47, \mathrm{XX}$ or $\mathrm{XY},+14$ \\
\hline $46, \mathrm{XX}, \mathrm{t}^{\mathrm{b}}$ & 33 & 3 & 0 & Success & \\
\hline $46, \mathrm{XX}, \mathrm{t}^{\mathrm{b}}$ & 35 & 3 & 0 & Success & \\
\hline $46, \mathrm{XX}, \mathrm{t}^{\mathrm{b}}$ & 42 & 4 & 0 & Not conceive & \\
\hline
\end{tabular}


Table 2 continued

\begin{tabular}{|c|c|c|c|c|c|}
\hline Reciprocal translocation & Age & $\begin{array}{l}\text { Previous } \\
\text { miscarriage } \\
\text { (Stillbirth) }\end{array}$ & $\begin{array}{l}\text { Previous live } \\
\text { birth }\end{array}$ & $\begin{array}{l}\text { Pregnancy } \\
\text { outcome }\end{array}$ & Chromosome \\
\hline \multicolumn{6}{|l|}{ Male } \\
\hline $46, X Y, t(1 ; 9)(q 42.3 ; q 22.3)$ & 35 & 3 & 0 & Not available & \\
\hline $46, X Y, t(1 ; 10)(p 32 ; q 26)$ & 31 & 4 & 0 & Success & \\
\hline $46, \mathrm{XY}, \mathrm{t}(1 ; 11)(\mathrm{p} 32.1 ; \mathrm{p} 15.1)$ & 33 & 2 & 0 & Success & \\
\hline 46,XY,t(2;7)(p10;q10) & 33 & 3 & 0 & Success & \\
\hline $46, \mathrm{XY}, \mathrm{t}(3 ; 5)(\mathrm{q} 26.2 ; \mathrm{p} 15.1)$ & 35 & 5 & 0 & Not available & \\
\hline $46, \mathrm{XY}, \mathrm{t}(3 ; 7)(\mathrm{q} 25.3 ; \mathrm{q} 21.1)$ & 31 & 4 & 0 & Success & \\
\hline $46, \mathrm{XY}, \mathrm{t}(3 ; 15)(\mathrm{p} 22 ; \mathrm{q} 26.2)$ & 35 & 3 & 0 & Success & \\
\hline $46, \mathrm{XY}, \mathrm{t}(4 ; 10)(\mathrm{p} 14 ; \mathrm{q} 21.2)$ & 42 & $2(1)$ & 0 & Not available & \\
\hline $46, X Y, t(4 ; 10)(\mathrm{q} 34 ; \mathrm{q} 21.2)$ & 29 & 2 & 0 & Success & \\
\hline 46,XY,t(5;6)(q33.1;p11.2) & 30 & 3 & 0 & Success & \\
\hline $46, X Y, t(5,9)^{b}$ & 32 & $2(2)$ & 1 & Success & \\
\hline $46, X Y, t(5 ; 10)(q 22 ; q 22)$ & 29 & 3 & 0 & Failure & Chemical $^{\mathrm{c}}$ \\
\hline $46, X Y, t(6 ; 14)(q 13 ; q 24), 15 p+$ & 36 & 3 & 0 & Not conceive & \\
\hline $46, X Y, t(6 ; 16)(q 27 ; p 13.1)$ & 31 & 3 & 0 & Success & \\
\hline $46, X Y, t(7 ; 8)(q 21 ; q 22)$ & 33 & 2 & 0 & Failure & $46, X X$ \\
\hline $46, X Y, t(7 ; 8)(q 32 ; q 22)$ & 25 & 2 & 0 & Failure & Chemical \\
\hline $46, X Y, t(7 ; 16)(p 22 ; q 21)$ & 35 & 3 & 0 & Not available & \\
\hline $46, X Y, t(7 ; 17)(q 11.23 ; q 23.3)$ & 25 & 5 & 0 & Success & \\
\hline $46, \mathrm{XY}, \mathrm{t}(8 ; 12)(\mathrm{p} 21.3 ; \mathrm{q} 12)$ & 31 & 4 & 1 & Success & \\
\hline $46, X Y, t(9 ; 13)(q 32 ; q 32), 46, X X, \operatorname{inv}(9)$ & 33 & 2 & 0 & Success & \\
\hline $46, X Y, t(10 ; 13)(q 24 ; q 34)$ & 28 & 3 & 1 & Success & \\
\hline $46, X Y, t(10 ; 16)(\mathrm{p} 14 ; \mathrm{q} 12.2)$ & 41 & 2 & 0 & PGD not conceive & \\
\hline $46, \mathrm{XY}, \mathrm{t}(11 ; 20)(\mathrm{q} 23.1 ; \mathrm{p} 13)$ & 25 & 3 & 0 & Not available & \\
\hline $46, X Y, t(11 ; 22)(\mathrm{q} 23.3 ; \mathrm{q} 11.2)$ & 30 & 3 & 1 & Success & \\
\hline $46, X Y, t(11 ; 22)(\mathrm{q} 24 ; \mathrm{q} 12), 46, \mathrm{XX}, \operatorname{inv}(9)$ & 33 & 3 & 0 & Success & \\
\hline 46,XY,t(13;17)(q14.1;q23) & 32 & 3 & 1 & Not available & \\
\hline $46, X Y, t(17 ; 21)(q 21 ; q 22)$ & 34 & 3 & 0 & Not conceive & \\
\hline $46, X Y, t^{b}$ & 33 & 2 & 0 & Failure & $46, X X$ \\
\hline
\end{tabular}

$P G D$ preimplantation genetic diagnosis

a These patients were not followed up after ascertainment of carrier status

b Details were unclear because these patients were examined in the previous hospital

${ }^{c}$ Chemical abortion

of miscarriages. The success rate might depend on the women's age, number of previous miscarriages, and the positions of breakpoints. Another reason is that the study concerned clinical data collected over 17 years. It is well known that patients with translocations sometimes miscarry despite a normal or balanced embryonic karyotype. The success rates for patients both with and without translocations in our study were superior to that in SugiuraOgasawara's earlier study because intervention methods such as anticoagulant and supportive psychotherapy might have now improved.

Cytogenetic analysis of semen from carrier men with translocations suggests that $46.9 \%$ exhibit alternate segregation in reciprocal translocation carriers and $88.7 \%$ with Robertsonian translocations (Gardner and Sutherland 2004). However, we cannot find who has difficulty in reaching successful delivery in RM patients with reciprocal translocations. For women with higher age or a high number of previous miscarriages, in vitro fertilization (IVF)-PGD might be able to save time and facilitate having a baby.

The live-birth rates with PGD per IVF in reciprocal translocation carriers $(23.7 \%, 47.2 \%$, and $6.2 \%)$ are comparable to or rather lower than those $(63.0 \%)$ with the subsequent first natural conception, as presented by this study (Chun et al. 2004; Otani et al. 2006; Feyereisen et al. 
Table 3 Carriers of a Robertsonian translocation with a history of recurrent miscarriage

$P G D$ preimplantation genetic diagnosis

a These patients were not followed up after ascertainment of carrier status

Table 4 Carriers of inversions and other abnormalities with a history of recurrent miscarriage

${ }^{a}$ These patients were not followed up after ascertainment of carrier status

b Normal variants

c Details were unclear because these patients were examined in the previous hospital

\begin{tabular}{|c|c|c|c|c|c|}
\hline Robertsonian translocation & Age & $\begin{array}{l}\text { Previous } \\
\text { miscarriage }\end{array}$ & $\begin{array}{l}\text { Previous } \\
\text { live birth }\end{array}$ & $\begin{array}{l}\text { Pregnancy } \\
\text { outcome }\end{array}$ & Chromosome \\
\hline \multicolumn{6}{|l|}{ Female } \\
\hline $\begin{array}{l}\text { 44,XX, der(13;22)(q10;q10), } \\
\quad \operatorname{der}(14 ; 15)(\mathrm{q} 10 ; \mathrm{q} 10)\end{array}$ & 33 & 3 & 0 & Success & \\
\hline 45,XX,der(13:14)(q10;q10) & 25 & 3 & 0 & Not available $^{a}$ & \\
\hline $45, X X, \operatorname{der}(13 ; 14)(q 10 ; q 10)$ & 33 & 3 & 0 & PGD on going & PGD \\
\hline $45, \mathrm{XX}, \operatorname{der}(13 ; 14)(\mathrm{q} 10 ; \mathrm{q} 14)$ & 32 & 3 & 0 & Not conceive & \\
\hline $45, \mathrm{XX}, \operatorname{der}(14 ; 14)(\mathrm{q} 10 ; \mathrm{q} 10)$ & 32 & 3 & 0 & Not available & \\
\hline $45, \mathrm{XX}, \operatorname{der}(14 ; 21)(\mathrm{q} 10 ; \mathrm{q} 10)$ & 33 & 3 & 0 & Not available & \\
\hline \multicolumn{6}{|l|}{ Male } \\
\hline $45, X Y, \operatorname{der}(13 ; 14)(q 10 ; q 10)$ & 32 & 2 & 1 & Failure & Not tested \\
\hline $45, \mathrm{XY}, \operatorname{der}(13 ; 14)(\mathrm{q} 10 ; \mathrm{q} 10)$ & 27 & 2 & 0 & PGD not available & \\
\hline $45, \mathrm{XY}, \operatorname{der}(13 ; 14)(\mathrm{q} 10 ; \mathrm{q} 10)$ & 30 & $2(1)$ & 1 & Failure & Not tested \\
\hline $45, \mathrm{XY}, \operatorname{der}(14 ; 21)(\mathrm{q} 10 ; \mathrm{q} 10)$ & 28 & 2 & 0 & Not available & \\
\hline $45, X Y, \operatorname{der}(15 ; 22)(q 10: q 10)$ & 28 & 3 & 0 & Not available & \\
\hline $45, X Y, \operatorname{der}(15 ; 22)(q 10 ; q 10)$ & 29 & 2 & 0 & Success & \\
\hline 45,XY,dic(13;14)(p11.2;p11.2) & 28 & 3 & 0 & Success & \\
\hline
\end{tabular}

\begin{tabular}{|c|c|c|c|c|c|}
\hline Other abnormalities & Age & $\begin{array}{l}\text { Previous } \\
\text { miscarriage }\end{array}$ & $\begin{array}{l}\text { Previous } \\
\text { live birth }\end{array}$ & $\begin{array}{l}\text { Pregnancy } \\
\text { outcome }\end{array}$ & Chromosome \\
\hline \multicolumn{6}{|l|}{ Female } \\
\hline $46, X X, \operatorname{ins}(8)(q 24.2 q 24.12 q 24.13)$ & 33 & 2 & 0 & Success & \\
\hline $46, X X, \operatorname{inv}(1)(\mathrm{p} 11 \mathrm{q} 21)$ & 31 & 2 & 0 & Success & \\
\hline 46,XX,inv(2)(p16q31) & 28 & 2 & 1 & Success & \\
\hline $46, X X, \operatorname{inv}(8)(\mathrm{p} 11.2 \mathrm{q} 22.1)$ & 33 & $2(1)$ & 0 & Not available $^{a}$ & \\
\hline $46, X X, \operatorname{inv}(11)(p 13 q 11)$ & 35 & 3 & 0 & Success & $46, X X$ \\
\hline $46, X X, \operatorname{inv}(17)(q 21.3 q 23)$ & 36 & 3 & 1 & Success & \\
\hline 46,X,del $(X)(q 25)$ & 24 & $3(1)$ & 0 & Success & \\
\hline $46, \mathrm{XX}, 19 \mathrm{cenh}+{ }^{\mathrm{b}}$ & 28 & 2 & 0 & Ectopy & \\
\hline $47, \mathrm{XX},+\mathrm{mar}$ & 37 & 2 & 0 & Failure & $47, \mathrm{XX},+22$ \\
\hline $47, \mathrm{XXX}$ & 36 & 10 & 0 & Not available & \\
\hline $47, \mathrm{XXX}$ & 30 & 3 & 0 & Not conceive & \\
\hline \multicolumn{6}{|l|}{ Male } \\
\hline $46, X Y, \operatorname{inv}(4)(q 12 q 21.3)$ & 43 & 2 & 1 & Not available & \\
\hline $46, X Y, \operatorname{inv}(7)^{\mathrm{c}}$ & 33 & 1 & 0 & Success & \\
\hline $46, X Y, \operatorname{inv}(8)^{c}$ & 29 & 2 & 0 & Failure & $46, X X$ \\
\hline $46, X Y, \operatorname{inv}(8)(\mathrm{p} 11.2 \mathrm{q} 24.1)$ & 28 & 3 & 1 & Failure & Not tested \\
\hline 46,XsmallY & 26 & 3 & 0 & Success & \\
\hline $47, \mathrm{XYY}$ & 35 & 2 & 0 & Success & \\
\hline $46, X Y, \operatorname{del}(16)^{\mathrm{c}}$ & 34 & 3 & 0 & Not available & \\
\hline
\end{tabular}

2007). It is difficult, however, to simply compare the superiority between IVF-PGD and natural conception in translocation carries, because information on the live-birth rate in the subsequent first pregnancy and time-based, not cycle-based, cumulative pregnancies after IVF-PGD or natural conception is very limited. Importantly, RM couples, not physicians and scientists, make the final decision; therefore, couples should be fully informed of advantages and disadvantages of both IVF-PGD and natural pregnancy. As the first step, we here report the outcome of subsequent first natural pregnancies in RM patients with translocation carries based on data obtained from multiple centers, which should be useful information for such couples. 
Acknowledgments We thank Dr. Hideto Yamada, Dr. Tatsuo Yamamoto, Dr. Osamu Ishihara, and Dr. Tatsuo Suzuki for their help.

\section{References}

Carp H, Feldman B, Oelsner G, Schiff E (2004) Parental karyotype and subsequent live births in recurrent miscarriage. Fertil Steril 81:1296-1301

De Braekeleer M, Dao TN (1990) Cytogenetic studies in couples experiencing repeated pregnancy losses. Hum Reprod 5:519-528

Elghezal H, Hider S, Braham R, Denguezli W, Ajina M, Saad A (2006) Chromosome abnormalities in one thousand infertile males with nonobstructive sperm disorders. Fertil Steril 86:1792-1795

Feyereisen E, Steffann J, Romana S, Lelorc 'Äôh M, Ray P, Kerbrat V, Tachdjian G, Frydman R, Frydman N (2007) Five years' experience of preimplantation genetic diagnosis in the Parisian Center: outcome of the first 441 started cycles. Fertil Steril 87:60-73

Franssen MTM, Korevaar JC, van der Veen F, Leschot NJ, Bossuyt PMM, Goddijn M (2006) Reproductive outcome after chromosome analysis in couples with two or more miscarriages: casecontrol study. BMJ 332:759-762

Gardner RJM, Sutherland GR (2004) Chromosome abnormalities and genetic counseling, 3rd edn. Oxford University Press, Oxford
Goddijn M, Joosten JH, Knegt AC, van der Veen F, Franssen MT, Bonsel GJ, Leschot NJ (2004) Clinical relevance of diagnosing structural chromosome abnormalities in couples with repeated miscarriage. Hum Reprod 19:1013-1017

Handyside AH, Kontogianni EH, Hardy K, Winston RM (1990) Pregnancies from biopsied human preimplantation embryos sexed by Y-specific DNA amplification. Nature 344:768-770

Kyu Lim C, Hyun Jun J, Mi Min D, Lee HS, Young Kim J, Koong MK, Kang IS (2004) Efficacy and clinical outcome of preimplantation genetic diagnosis using FISH for couples of reciprocal and Robertsonian translocations: the Korean experience. Prenat Diagn 24:556-561

Munne S, Sandalinas M, Escudero T, Fung J, Gianaroli L, Cohen J (2000) Outcome of preimplantation genetic diagnosis of translocations. Fertil Steril 73:1209-1218

Otani T, Roche M, Mizuike M, Colls P, Escudero T, Munne S (2006) Preimplantation genetic diagnosis significantly improves the pregnancy outcome of translocation carriers with a history of recurrent miscarriage and unsuccessful pregnancies. Reprod Biomed Online 13:869-874

Stephenson MD, Sierra S (2006) Reproductive outcomes in recurrent pregnancy loss associated with a parental carrier of a structural chromosome rearrangement. Hum Reprod 21:1076-1082

Sugiura-Ogasawara M, Ozaki Y, Sato T, Suzumori N, Suzumori K (2004) Poor prognosis of recurrent aborters with either maternal or paternal reciprocal translocations. Fertil Steril 81:367-373 